\title{
Existence, Uniqueness and Data Dependence for the Solutions of some Integro-Differential Equations of Mixed Type in Banach Space
}

\author{
V. Mureşan
}

\begin{abstract}
In this paper we study existence, uniqueness and data dependence for the solutions of some integro-differential equations of mixed type in Banach space by using Picard and weakly Picard operators' technique and suitable Bielecki norms.

Keywords: Integro-differential equations, fixed points, Picard operators, weakly Picard operators
\end{abstract}

AMS subject classification: $34 \mathrm{~K} 05,47 \mathrm{H} 10$

\section{Introduction}

Ordinary differential equations, functional differential equations with or without deviating argument and equations in abstract spaces have been studied in many papers. In the papers $[3,6]$ theorems about the existence and uniqueness of solutions of some abstract nonlinear non-local Cauchy problems in Banach spaces were considered and in the paper [4] a theorem about the existence of an approximate solution to an abstract nonlinear non-local Cauchy problem in a Banach space was given, too. We remark in the same field the monographs [5, 9, 11 - 13].

Integro-differential equations of mixed type in Banach spaces have been studied in the papers [7, 10], and integro-differential equations of mixed type with impulses in Banach spaces were considered in the paper [14], too. Fredholm-Volterra integral equations in relationship with Maia's theorem were considered in the paper [16].

The aim of the present paper is to obtain existence, uniqueness and data dependence results for the solutions of some integro-differential equations of

V. Mureşan: Techn. Univ. of Cluj-Napoca, Dept. Math., C. Daicoviciu str. 15, 3400 Cluj-Napoca, Romania; vmuresan@math.utcluj.ro

ISSN 0232-2064 / \$2.50 C Heldermann Verlag Berlin 
mixed type in Banach space. To do this we use Picard and weakly Picard operators' technique due to I. A. Rus (see [18 - 22]). So, our technique is different from those used in the papers quoted above.

Let $(X,\|\cdot\|)$ be a Banach space. Consider the problem

$$
\left.\begin{array}{rl}
x^{\prime}(t) & =f\left(t, x(t), \int_{0}^{t} K_{1}(t, s) x(s) d s, \int_{0}^{T} K_{2}(t, s) x(s) d s\right) \\
x(0) & =x_{0}
\end{array}\right\}
$$

on $[0, T]$, where $f \in C\left([0, T] \times X^{3}, X\right), K_{i} \in C\left(D_{i}, \mathbb{R}\right) \quad(i=1,2)$ and $x_{0} \in X$. Here

$$
\begin{aligned}
& D_{1}=\left\{(t, s) \in \mathbb{R}^{2}: 0 \leq s \leq t \leq T\right\} \\
& D_{2}=[0, T] \times[0, T] .
\end{aligned}
$$

It is well known that $x \in C^{1}([0, T], X)$ is a solution of problem (1) if and only if $x$ is a solution in $C([0, T], X)$ of the integro-differential equation

$$
x(t)=x_{0}+\int_{0}^{t} f\left(\xi, x(\xi), \int_{0}^{\xi} K_{1}(\xi, s) x(s) d s, \int_{0}^{T} K_{2}(\xi, s) x(s) d s\right) d \xi
$$

on $[0, T]$.

In [10] the author combines topological degree theory and monotone iterative technique given in [12] to investigate the existence of solutions and also minimal and maximal solutions of problem (1). In the present paper we consider suitable Bielecki norms in a convenient space and obtain existence, uniqueness and data dependence results for the solutions of equation (2) which is equivalent to problem (1).

In [7] the authors study the existence of solutions of the abstract non-local integro-differential Cauchy problem in arbitrary Banach spaces

$$
\left.\begin{array}{rl}
x^{\prime}(t) & =f\left(t, x(t), \int_{0}^{t} K_{1}(t, s) x(s) d s, \int_{0}^{T} K_{2}(t, s) x(s) d s\right) \\
x(0) & =x_{0}-\sum_{i=1}^{p} c_{i} x\left(t_{i}\right)
\end{array}\right\}
$$

on $[0, T]$, where $f \in C\left([0, T] \times X^{3}, X\right), 0<t_{1}<t_{2}<\ldots<t_{p} \leq T, c_{i} \neq 0$, $p \in \mathbb{N}$ and $x_{0} \in X$. This problem is equivalent to the integro-differential equation

$$
\begin{aligned}
x(t)= & x_{0}-\sum_{i=1}^{p} c_{i} x\left(t_{i}\right) \\
& +\int_{0}^{t} f\left(\xi, x(\xi), \int_{0}^{\xi} K_{1}(\xi, s) x(s) d s, \int_{0}^{T} K_{2}(\xi, s) x(s) d s\right) d \xi
\end{aligned}
$$


on $[0, T]$. For this purpose, the Kuratowski measure of non-compactness, fixed point principles and a monotone iterative technique were applied. We remark that the weakly Picard operators technique can be used to prove existence of solutions to equation (3).

\section{Preliminaries}

Let $(X, d)$ be a metric space and $A: X \rightarrow X$ an operator. We shall use the following notations:

$$
\begin{aligned}
& P(X)=\{Y \subseteq X \mid Y \neq \emptyset\} \\
& F_{A}=\{x \in X \mid A(x)=x\}-\text { the fixed point set of } A \\
& I(A)=\{Y \in P(X) \mid A(Y) \subseteq Y\} \\
& O_{A}(x)=\left\{x, A(x), A^{2}(x), \ldots, A^{n}(x), \ldots\right\} \text { - the } A \text {-orbit of } x \in X \\
& H: P(X) \times P(X) \rightarrow \mathbb{R}_{+} \cup\{+\infty\} \\
& H(Y, Z)=\max \left(\sup _{a \in Y} \inf _{b \in Z} d(a, b), \sup _{b \in Z} \inf _{a \in Y} d(a, b)\right) \\
& \quad \text { - the Pompeiu-Hausdorff functional on } P(X) .
\end{aligned}
$$

Definition 2.1 (Rus [18]). Let $(X, d)$ be a metric space. An operator $A: X \rightarrow X$ is a Picard operator if there exists $x^{*} \in X$ such that $F_{A}=\left\{x^{*}\right\}$ and the sequence $\left(A^{n}\left(x_{0}\right)\right)_{n \in \mathbb{N}}$ converges to $x^{*}$ for all $x_{0} \in X$.

Definition 2.2 (Rus [19]). Let $(X, d)$ be a metric space. An operator $A: X \rightarrow X$ is a weakly Picard operator if the sequence $\left(A^{n}\left(x_{0}\right)\right)_{n \in \mathbb{N}}$ converges for all $x_{0} \in X$ and its limit (which may depend on $x_{0}$ ) is a fixed point of $A$.

If $A$ is a weakly Picard operator, then we consider the operator

$$
A^{\infty}: X \rightarrow X, \quad A^{\infty}(x)=\lim _{n \rightarrow \infty} A^{n}(x)
$$

The following results are useful in what follows:

Theorem 2.1 [17]. Let $(Y, d)$ be a complete metric space and $A, B: Y \rightarrow$ $Y$ two operators. We suppose the following:

(i) $A$ is a contraction with contraction constant $\alpha$ and $F_{A}=\left\{x_{A}^{*}\right\}$.

(ii) $B$ has fixed points and $x_{B}^{*} \in F_{B}$.

(iii) There exists $\eta>0$ such that $d(A(x), B(x)) \leq \eta$, for all $x \in Y$. Then $d\left(x_{A}^{*}, x_{B}^{*}\right) \leq \frac{\eta}{1-\alpha}$.

Theorem $2.2[22]$. Let $(X, d)$ be a complete metric space and $A, B$ : $X \rightarrow X$ two orbitally continuous operators. We suppose the following: 
(i) There exists $\alpha \in[0,1)$ such that

$$
\begin{aligned}
& d\left(A^{2}(x), A(x)\right) \leq \alpha d(x, A(x)) \\
& d\left(B^{2}(x), B(x)\right) \leq \alpha d(x, B(x))
\end{aligned} \quad(x \in X) .
$$

(ii) There exists $\eta>0$ such that $d(A(x), B(x)) \leq \eta$ for all $x \in X$.

Then $H\left(F_{A}, F_{B}\right) \leq \frac{\eta}{1-\alpha}$ where $H$ denotes the Pompeiu-Hausdorff functional.

Theorem 2.3 [19]. Let $(X, d)$ be a metric space. Then $A: X \rightarrow X$ is a weakly Picard operator if and only if there exists a partition $X=\bigcup_{\lambda \in \Lambda} X_{\lambda}$ of $X$ such that

(a) $X_{\lambda} \in I(A)$

(b) $\left.A\right|_{X_{\lambda}}: X_{\lambda} \rightarrow X_{\lambda}$ is a Picard operator, for all $\lambda \in \Lambda$.

Consider a Banach space $(X,\|\cdot\|)$, let $\|\cdot\|_{B}$ and $\|\cdot\|_{C}$ be the Bielecki and Chebyshev norms on $C([0, T], X)$ defined by

$$
\|x\|_{B}=\max _{t \in[0, T]}\|x(t)\| e^{-\tau t} \quad(\tau>0) \quad \text { and } \quad\|x\|_{C}=\max _{t \in[0, T]}\|x(t)\|
$$

and denote by $d_{B}$ and $d_{C}$ their corresponding metrics. We consider the set

$$
C_{L}([0, T], X)=\left\{\begin{array}{ll}
x \in C([0, T], X) \mid \begin{array}{l}
\left\|x\left(t_{1}\right)-x\left(t_{2}\right)\right\| \leq L\left|t_{1}-t_{2}\right| \\
\text { for all } t_{1}, t_{2} \in[0, T]
\end{array}
\end{array}\right\}
$$

where $L>0$ and $B_{R}=\{x \in X:\|x\| \leq R\}$ with $R>0$. If $d \in\left\{d_{C}, d_{B}\right\}$, then $(C([0, T], X), d)$ and $\left(C_{L}([0, T], X), d\right)$ are complete metric spaces.

\section{A integro-differential equation of mixed type}

Consider equation (2). Denote $k_{i}=\max _{(t, s) \in D_{i}}\left|K_{i}(t, s)\right| \quad(i=1,2)$. We have

Theorem 3.1. Suppose the following:

(i) $f \in C\left([0, T] \times X^{3}, X\right)$.

(ii) There exists a constant $M>0$ such that $\|f(s, u, v, w)\| \leq M$ for all $u, v, w \in X$ and all $s \in[0, T]$.

(iii) $M \leq L$.

(iv) There exists a constant $L_{0}>0$ such that

$$
\begin{aligned}
\| f\left(s, u_{1}, v_{1}, w_{1}\right) & -f\left(s, u_{2}, v_{2}, w_{2}\right) \| \\
\leq & L_{0}\left(\left\|u_{1}-u_{2}\right\|+\left\|v_{1}-v_{2}\right\|+\left\|w_{1}-w_{2}\right\|\right)
\end{aligned}
$$


for all $u_{i}, v_{i}, w_{i} \in X \quad(i=1,2)$ and all $s \in[0, T]$.

(v) There exists a constant $\tau>0$ such that $\frac{L_{0}}{\tau}\left(1+\frac{k_{1}}{\tau}+k_{2} T e^{\tau T}\right)<1$.

Then equation (2) has a unique solution $x^{*}$ in $C_{L}([0, T], X)$, and this solution can be obtained by the successive approximation method, starting from any element of $C_{L}([0, T], X)$.

Proof. Consider the continuous operator

$$
A:\left(C_{L}([0, T], X),\|\cdot\|_{B}\right) \rightarrow\left(C_{L}([0, T], X),\|\cdot\|_{B}\right)
$$

defined by

$$
\begin{aligned}
& A(x)(t) \\
& =x_{0}+\int_{0}^{t} f\left(\xi, x(\xi), \int_{0}^{\xi} K_{1}(\xi, s) x(s) d s, \int_{0}^{T} K_{2}(\xi, s) x(s) d s\right) d \xi
\end{aligned}
$$

We have

$$
\begin{aligned}
\|A(x)(t)-A(z)(t)\| & \int_{0}^{t} \| f\left(\xi, x(\xi), \int_{0}^{\xi} K_{1}(\xi, s) x(s) d s, \int_{0}^{T} K_{2}(\xi, s) x(s) d s\right) \\
\leq & f\left(\xi, z(\xi), \int_{0}^{\xi} K_{1}(\xi, s) z(s) d s, \int_{0}^{T} K_{2}(\xi, s) z(s) d s\right) \| d \xi \\
\leq & L_{0} \int_{0}^{t}\left[\|x(\xi)-z(\xi)\|+\left\|\int_{0}^{\xi} K_{1}(\xi, s)(x(s)-z(s)) d s\right\|\right. \\
+ & \left.\left\|\int_{0}^{T} K_{2}(\xi, s)(x(s)-z(s)) d s\right\|\right] d \xi \\
\leq & L_{0}\left[\int_{0}^{t}\|x(\xi)-z(\xi)\| d \xi+k_{1} \int_{0}^{t}\left(\int_{0}^{\xi}\|x(s)-z(s)\| d s\right) d \xi\right. \\
+ & \left.k_{2} \int_{0}^{t}\left(\int_{0}^{T}\|x(s)-z(s)\| d s\right) d \xi\right] \\
\leq & L_{0}\left[\int_{0}^{t}\|x(\xi)-z(\xi)\| e^{-\tau \xi} e^{\tau \xi} d \xi\right. \\
+ & k_{1} \int_{0}^{t}\left(\int_{0}^{\xi}\|x(s)-z(s)\| e^{-\tau s} e^{\tau s} d s\right) d \xi \\
+ & \left.k_{2} \int_{0}^{t}\left(\int_{0}^{T}\|x(s)-z(s)\| e^{-\tau s} e^{\tau s} d s\right) d \xi\right]
\end{aligned}
$$




$$
\begin{aligned}
\leq & L_{0}\|x-z\|_{B}\left[\int_{0}^{t} e^{\tau \xi} d \xi+k_{1} \int_{0}^{t}\left(\int_{0}^{\xi} e^{\tau s} d s\right) d \xi\right. \\
& \left.+k_{2} \int_{0}^{t}\left(\int_{0}^{T} e^{\tau s} d s\right) d \xi\right] \\
= & L_{0}\|x-z\|_{B}\left[\left(\frac{e^{\tau t}}{\tau}-\frac{1}{\tau}\right)+k_{1} \int_{0}^{t}\left(\frac{e^{\tau \xi}}{\tau}-\frac{1}{\tau}\right) d \xi\right. \\
& \left.+k_{2} \int_{0}^{t}\left(\frac{e^{\tau T}}{\tau}-\frac{1}{\tau}\right) d \xi\right] \\
= & L_{0}\|x-z\|_{B}\left[\left(\frac{e^{\tau t}}{\tau}-\frac{1}{\tau}\right)+\frac{k_{1}}{\tau}\left(\frac{e^{\tau t}}{\tau}-\frac{1}{\tau}-t\right)+\frac{k_{2}}{\tau}\left(e^{\tau T}-1\right) t\right] \\
\leq & L_{0}\|x-z\|_{B}\left[\frac{e^{\tau t}}{\tau}+\frac{k_{1}}{\tau} \frac{e^{\tau t}}{\tau}+k_{2} \frac{e^{\tau t}}{\tau} e^{\tau(T-t)} T\right] \\
\leq & L_{0} \frac{1}{\tau} e^{\tau t}\left(1+\frac{k_{1}}{\tau}+k_{2} T e^{\tau T}\right)\|x-z\|_{B}
\end{aligned}
$$

for all $x, z \in C_{L}([0, T], X)$. It follows that

$$
\|A(x)(t)-A(z)(t)\| e^{-\tau t} \leq \frac{L_{0}}{\tau}\left(1+\frac{k_{1}}{\tau}+k_{2} T e^{\tau T}\right)\|x-z\|_{B}
$$

for all $t \in[0, T]$. So

$$
\|A(x)-A(z)\|_{B} \leq \frac{L_{0}}{\tau}\left(1+\frac{k_{1}}{\tau}+k_{2} T e^{\tau T}\right)\|x-z\|_{B}
$$

for all $x, z \in C_{L}([0, T], X)$. The operator $A$ is of Lipschitz type with constant

$$
L_{A}=\frac{L_{0}\left(1+\frac{k_{1}}{\tau}+k_{2} T e^{\tau T}\right)}{\tau}
$$

and $0<L_{A}<1$. By applying the Contraction Principle to this operator we obtain that $A$ is a Picard operator

Similarly as above, we can prove

Theorem 3.2. Suppose the following:

(i) $f \in C\left([0, T] \times B_{R}^{3}, X\right)$ with $\|f(s, u, v, w)\| \leq M(R)$ for all $s \in[0, T]$ and $u, v, w \in B_{R}$.

(ii) $M(R) \leq L$.

(iii) $k_{i} T \leq 1 \quad(i=1,2)$.

(iv) $\left\|x_{0}\right\|+M(R) T \leq R$. 
(v) There exists a constant $L_{0}>0$ such that

$$
\begin{aligned}
\| f\left(s, u_{1}, v_{1}, w_{1}\right) & -f\left(s, u_{2}, v_{2}, w_{2}\right) \| \\
\leq & L_{0}\left(\left\|u_{1}-u_{2}\right\|+\left\|v_{1}-v_{2}\right\|+\left\|w_{1}-w_{2}\right\|\right)
\end{aligned}
$$

for all $u_{i}, v_{i}, w_{i} \in B_{R} \quad(i=1,2)$ and all $s \in[0, T]$.

(vi) There exists a constant $\tau>0$ such that $\frac{L_{0}}{\tau}\left(1+\frac{k_{1}}{\tau}+k_{2} T e^{\tau T}\right)<1$.

Then equation (2) has a unique solution in $C_{L}\left([0, T], B_{R}\right)$, and this solution can be obtained by the successive approximation method, starting from any element of $C_{L}\left([0, T], B_{R}\right)$.

Remark 3.1. If we consider the problem

$$
\left.\begin{array}{rl}
x^{\prime}(t) & =\frac{1}{10} \int_{0}^{t} \sin (t+s) x(s) d s+\frac{1}{18} \int_{0}^{\frac{1}{3}} \cos (t s) x(s) d s \\
x(0) & =0
\end{array}\right\}
$$

on $[0, T]$, then $L_{0}=1, k_{1}=\frac{1}{10}, k_{2}=\frac{1}{18}$, and for $\tau=2$ we have condition (vi) in Theorem 3.2.

Now, we consider both equation (2) and

$$
x(t)=y_{0}+\int_{0}^{t} g\left(\xi, x(\xi), \int_{0}^{\xi} K_{1}(\xi, s) x(s) d s, \int_{0}^{T} K_{2}(\xi, s) x(s) d s\right) d \xi
$$

on $[0, T]$, where $g \in C\left([0, T] \times X^{3}, X\right)$ and $K_{i} \in C\left(D_{i}, \mathbb{R}\right) \quad(i=1,2)$ are the same as in equation (2) and $y_{0} \in X$. We have

Theorem 3.3. Suppose the following:

(i) All conditions in Theorem 3.1 are satisfied and $x^{*} \in C_{L}([0, T], X)$ is the unique solution of equation (2).

(ii) There exists a constant $M_{1}>0$ such that $\|g(s, u, v, w)\| \leq M_{1}$ for all $u, v, w \in X$ and all $s \in[0, T]$.

(iii) With the same Lipschitz constant $L_{0}$ as in Theorem 3.1,

$$
\begin{aligned}
\| g\left(s, u_{1}, v_{1}, w_{1}\right) & -g\left(s, u_{2}, v_{2}, w_{2}\right) \| \\
\leq & L_{0}\left(\left\|u_{1}-u_{2}\right\|+\left\|v_{1}-v_{2}\right\|+\left\|w_{1}-w_{2}\right\|\right)
\end{aligned}
$$

for all $u_{i}, v_{i}, w_{i} \in X \quad(i=1,2)$ and all $s \in[0, T]$.

(iv) $M_{1} \leq L$.

(v) There exists a constant $\eta>0$ such that

$$
\|f(s, u, v, w)-g(s, u, v, w)\| \leq \eta
$$


for all $u, v, w \in X$ and $s \in[0, T]$.

Then, if $y^{*}$ is the solution of equation (5),

$$
\left\|x^{*}-y^{*}\right\|_{B} \leq \frac{\left\|x_{0}-y_{0}\right\|+\eta T}{1-L_{A}}
$$

where $L_{A}$ is given by (4) with $\tau=\tau_{0}>0$ such that $0<L_{A}<1$.

Proof. Consider the operators

$$
A, B: C_{L}([0, T], X) \rightarrow C_{L}([0, T], X)
$$

defined by

$$
\begin{aligned}
& A(x)(t)=x_{0}+\int_{0}^{t} f\left(\xi, x(\xi), \int_{0}^{\xi} K_{1}(\xi, s) x(s) d s, \int_{0}^{T} K_{2}(\xi, s) x(s) d s\right) d \xi \\
& B(x)(t)=y_{0}+\int_{0}^{t} g\left(\xi, x(\xi), \int_{0}^{\xi} K_{1}(\xi, s) x(s) d s, \int_{0}^{T} K_{2}(\xi, s) x(s) d s\right) d \xi
\end{aligned}
$$

on $[0, T]$, in which $K_{i} \in C\left(D_{i}, \mathbb{R}\right) \quad(i=1,2)$ are the same. We have

$$
\|A(x)(t)-B(x)(t)\| \leq\left\|x_{0}-y_{0}\right\|+\eta T \quad(t \in[0, T]) .
$$

It follows that

$$
\|A(x)-B(x)\|_{B} \leq\left\|x_{0}-y_{0}\right\|+\eta T .
$$

So we can apply Theorem 2.1

Remark 3.2. The results obtained in this section can be generalized to study existence, uniqueness and data dependence for the solutions of the problem with linear modification of the argument

$$
\left.\begin{array}{rl}
x^{\prime}(t) & =f\left(t, x(t), x(\lambda t), \int_{0}^{t} K_{1}(t, s) x(\lambda s) d s, \int_{0}^{T} K_{2}(t, s) x(\lambda s) d s\right) \\
x(0) & =x_{0}
\end{array}\right\}
$$

on $[0, T]$, where $0<\lambda<1, f \in C\left([0, T] \times X^{4}, X\right), K_{i} \in C\left(D_{i}, R\right) \quad(i=1,2)$ and $x_{0} \in X$. This problem is more general than those considered in [15]. 


\section{Another integro-differential equation of mixed type}

Now, we consider the integral equation of mixed type

$$
x(t)=x(0)+\int_{0}^{t} f\left(\xi, x(\xi), \int_{0}^{\xi} K_{1}(\xi, s) x(s) d s, \int_{0}^{T} K_{2}(\xi, s) x(s) d s\right) d \xi
$$

on $[0, T]$, where $f \in C\left([0, T] \times X^{3}, X\right), K_{i} \in C\left(D_{i}, \mathbb{R}\right)$ and $D_{i} \quad(i=1,2)$ are as in problem (1). We have

Theorem 4.1. Suppose that for equation (6) the same conditions as in Theorem 3.1 are satisfied. Then this equation has solutions in $C_{L}([0, T], X)$. If $S \subset C_{L}([0, T], X)$ is its solutions set, then $\operatorname{card} S=\operatorname{card} X$.

Proof. Consider the operator

$$
A_{*}: C_{L}([0, T], X) \rightarrow C_{L}([0, T], X)
$$

defined by

$$
\begin{aligned}
& A_{*}(x)(t) \\
& =x(0)+\int_{0}^{t} f\left(\xi, x(\xi), \int_{0}^{\xi} K_{1}(\xi, s) x(s) d s, \int_{0}^{T} K_{2}(\xi, s) x(s) d s\right) d \xi .
\end{aligned}
$$

This is a continuous operator, but not a Lipschitz one. We can write

$$
C_{L}([0, T], X)=\bigcup_{\alpha \in X} X_{\alpha}, \quad X_{\alpha}=\left\{x \in C_{L}([0, T], X): x(0)=\alpha\right\} .
$$

We have that $X_{\alpha}$ is an invariant set of $A_{*}$ and we apply Theorem 3.1 to $\left.A_{*}\right|_{X_{\alpha}}$. By using Theorem 2.3 we obtain that $A_{*}$ is a weakly Picard operator. Consider the operator

$$
A_{*}^{\infty}: C_{L}([0, T], X) \rightarrow C_{L}([0, T], X), \quad A_{*}^{\infty}(x)=\lim _{n \rightarrow \infty} A_{*}^{n}(x) .
$$

From $A_{*}^{n+1}(x)=A_{*}\left(A_{*}^{n}(x)\right)$ and the continuity of $A_{*}, A_{*}^{\infty}(x) \in F_{A_{*}}$. Then $A_{*}^{\infty}\left(C_{L}([0, T], X)\right)=F_{A_{*}}=S$, and $S \neq \emptyset$. So, $\operatorname{card} S=\operatorname{card} X$

Remark 4.1. Similarly as above we can prove the existence of solutions of equation (3) that corresponds to a problem considered in [7].

In order to study data dependence for the solutions set of equation (6) we consider both (6) and the equation

$$
x(t)=x(0)+\int_{0}^{t} g\left(\xi, x(\xi), \int_{0}^{\xi} K_{1}(\xi, s) x(s) d s, \int_{0}^{T} K_{2}(\xi, s) x(s) d s\right) d \xi
$$

on $[0, T]$ where $K_{1}, K_{2}$ are the same as in (6) and $g \in C\left([0, T] \times X^{3}, X\right)$. Let $S_{1}$ be the solutions set of this equation. 
Theorem 4.2. Suppose the following:

(i) There exists a constant $L_{*}>0$ such that

$$
\begin{aligned}
\| f\left(s, u_{1}, v_{1}, w_{1}\right) & -f\left(s, u_{2}, v_{2}, w_{2}\right) \| \\
\leq & L_{*}\left(\left\|u_{1}-u_{2}\right\|+\left\|v_{1}-v_{2}\right\|+\left\|w_{1}-w_{2}\right\|\right) \\
\| g\left(s, u_{1}, v_{1}, w_{1}\right) & -g\left(s, u_{2}, v_{2}, w_{2}\right) \| \\
\leq & L_{*}\left(\left\|u_{1}-u_{2}\right\|+\left\|v_{1}-v_{2}\right\|+\left\|w_{1}-w_{2}\right\|\right)
\end{aligned}
$$

for all $u_{i}, v_{i}, w_{i} \in X \quad(i=1,2)$ and all $s \in[0, T]$.

(ii) There exists a constant $M_{*}>0$ such that

$$
\begin{aligned}
\|f(s, u, v, w)\| & \leq M_{*} \\
\|g(s, u, v, w)\| & \leq M_{*}
\end{aligned}
$$

for all $u, v, w \in X$ and all $s \in[0, T]$.

(iii) $M_{*} \leq L_{*}$.

(iv) There exists a constant $\eta_{1}>0$ such that

$$
\|f(s, u, v, w)-g(s, u, v, w)\| \leq \eta_{1}
$$

for all $u, v, w \in X$ and all $s \in[0, T]$.

(v) $3 L_{*} T k_{0}<1$, where $k_{0}=\max \left(1, k_{1} T, k_{2} T\right)$.

Then

$$
H_{\|\cdot\|_{C}}\left(S, S_{1}\right) \leq \frac{\eta_{1} T}{1-3 L_{*} T k_{0}}
$$

where by $H_{\|\cdot\|_{C}}$ we denote the Pompeiu-Hausdorff functional with respect to $\|\cdot\|_{C}$ on $C_{L}([0, T], X)$.

Proof. Consider the operator

$$
B_{*}: C_{L}([0, T], X) \rightarrow C_{L}([0, T], X)
$$

defined by

$$
B_{*}(x)(t)=x(0)+\int_{0}^{t} g\left(\xi, x(\xi), \int_{0}^{\xi} K_{1}(\xi, s) x(s) d s, \int_{0}^{T} K_{2}(\xi, s) x(s) d s\right) d \xi
$$

on $[0, T]$. We have

$$
\begin{aligned}
\| A_{*}^{2}(x)(t) & -A_{*}(x)(t) \| \\
& \leq L_{*} \int_{0}^{t}\left[\left\|A_{*}(x)(\xi)-x(\xi)\right\|\right.
\end{aligned}
$$




$$
\begin{aligned}
& +\left\|\int_{0}^{\xi} K_{1}(\xi, s)\left(A_{*}(x)(s)-x(s)\right) d s\right\| \\
& \left.+\left\|\int_{0}^{T} K_{2}(\xi, s)\left(A_{*}(x)(s)-x(s)\right) d s\right\|\right] d \xi \\
& \leq 3 L_{*} T \max \left(1, k_{1} T, k_{2} T\right)\left\|A_{*}(x)-x\right\|_{C} \\
& =3 L_{*} T k_{0}\left\|A_{*}(x)-x\right\|_{C}
\end{aligned}
$$

for all $x \in C_{L}([0, T], X)$. Similarly,

$$
\left\|B_{*}^{2}(x)(t)-B_{*}(x)(t)\right\| \leq 3 L_{*} T k_{0}\left\|B_{*}(x)-x\right\|_{C}
$$

for all $x \in C_{L}([0, T], X)$. It follows that

$$
\begin{gathered}
\left\|A_{*}^{2}(x)-A_{*}(x)\right\|_{C} \leq 3 L_{*} T k_{0}\left\|A_{*}(x)-x\right\|_{C} \\
\left\|B_{*}^{2}(x)-B_{*}(x)\right\|_{C} \leq 3 L_{*} T k_{0}\left\|B_{*}(x)-x\right\|_{C} .
\end{gathered}
$$

Because of assumption (iv), $\left\|A_{*}(x)-B_{*}(x)\right\|_{C} \leq \eta_{1} T$ for all $x \in C_{L}([0, T], X)$. By applying Theorem 2.2 we obtain $H_{\|\cdot\|_{C}}\left(F_{A_{*}}, F_{B_{*}}\right) \leq \frac{\eta_{1} T}{1-3 L_{*} T k_{0}}$ and the theorem is proved

\section{References}

[1] Aman, H.: On the number of solutions of nonlinear equations in ordered Banach spaces. J. Funct. Anal. 11 (1972), 346 - 384.

[2] Buică, A.: Existence and continuous dependence of solutions of some functionaldifferential equations. Preprint. Cluj-Napoca: Babeş-Bolyai Univ. (Romania), Seminar on Fixed Point Theory, Preprint 3 (1995), 1 - 13.

[3] Byszewski, L.: Theorems about the existence and uniqueness of solutions of a semilinear evolution nonlocal Cauchy problem. J. Math. Anal. Appl. 162 (1991), $494-505$.

[4] Byszewski, L.: Existence of approximate solution to abstract nonlocal Cauchy problem. J. Appl. Math. \& Stoch. Anal. 5 (1992), 363 - 374.

[5] Byszewski, L.: Differential and Functional-Differential Problems with Nonlocal Conditions (in Polish; Cracow Univ. Techn. Monograph: Vol. 184). Cracow: Univ. Techn. 1995.

[6] Byszewski, L. and V. Lakshmikantham: Theorem about the existence and uniqueness of a solution of a nonlocal abstract Cauchy problem in a Banach space. Appl. Anal. 40 (1991), $11-19$.

[7] Byszewski, L. and A. Tabor: An application of the Kuratowski measure of noncompactness to an investigation of the existence of solutions of an abstract integrodifferential problem. Nonlin. Studies 6 (1999), $111-122$. 
[8] Du, S. W. and V. Lakshmikantham: Monotone iterative technique for differential equations in a Banach space. J. Math. Anal. Appl. 87 (1982), $454-$ 459.

[9] Guo Dajun: Ordinary Differential Equations in Abstract Spaces (in Chinese) Jiuan Shandong Sci. and Tech. Press 1985.

[10] Guo Dajun: Solutions of nonlinear integrodifferential equations of mixed type in Banach spaces. J. Appl. Math. Simul. 2 (1989), 1 - 11.

[11] Guo Dajun and V. Lakshmikantham: Nonlinear Problems in Abstract Cones. Boston - New York: Acad. Press 1988.

[12] Lakshmikantham, V. and S. Leela: Nonlinear Differential Equations in Abstract Spaces. Oxford et al.: Pergamon Press 1981.

[13] Lakshmikantham, V., Bainov, D. D. and P. S. Simeonov: Theory of Impulsive Differential Equations. Singapore: World Science 1989.

[14] Liu Wei-an: Solutions of nonlinear integrodifferential equations of mixed type with impulses in Banach spaces. Math. Aplicata, Jingyong Shuxue Math. Appl. 9 (1996), 58 - 62.

[15] Mureşan, V.: Differential Equations with Affine Modification of the Argument (in Romania). Cluj-Napoca (Romania): Transilvania Press 1997.

[16] Petruşel, A.: Fredholm-Volterra integral equations and Maia's theorem. Preprint. Cluj-Napoca (Romania): Babeş-Boyai Univ., Seminar on Fixed Point Theory, Preprint 3 (1988), $79-82$.

[17] Rus,I. A.: Metrical Fixed Point Theorems. Univ. of Cluj-Napoca (Romania) 1979.

[18] Rus, I. A.: Picard mappings: results and problems. Preprint. Cluj-Napoca: Babeş-Bolyai Univ., Seminar on Fixed Point Theory, Preprint 6 (1987), 55 64.

[19] Rus, I. A.: Weakly Picard mappings. Comment. Math. Univ. Carolinae 34 (1993), $769-773$.

[20] Rus, I. A.: Picard operators and applications. Preprint. Cluj-Napoca (Romania): Babeş-Bolyai Univ., Seminar on Fixed Point Theory, Preprint 3 (1996).

[21] Rus, I. A.: Weakly Picard operators and applications. Cluj-Napoca (Romania): Babeş-Bolyai Univ., Seminar on Fixed Point Theory 2 (2000), 41 - 57.

[22] Rus, I. A. and S. Mureşan: Data dependence of the fixed points set of some weakly Picard operators. In: Proc. Itinerant Seminar (Elena Popoviciu editor, Srima Publishing House), Cluj-Napoca (Romania): Babeş-Bolyai Univ. 2000, pp. $201-207$.

Received 04.04.2003, in revised form 16.12.2003 